\title{
Evaluation of Cardiac Findings in Mucopolysaccharidosis Type III Patients
}

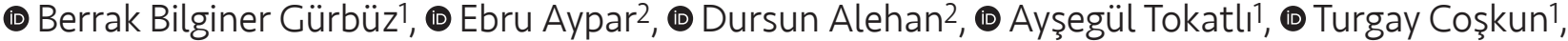 \\ (D) Ali Dursun1, (1) H. Serap Sivri1
}

${ }^{1}$ Hacettepe University Faculty of Medicine, Department of Pediatric Metabolism, Ankara, Turkey

${ }^{2}$ Hacettepe University Faculty of Medicine, Department of Pediatric Cardiology, Ankara, Turkey

\begin{abstract}
Aim: To investigate cardiac involvement in patients diagnosed with mucopolysaccharidosis type III (MPS III) in a university hospital in Turkey. Materials and Methods: This descriptive cross-sectional study was performed in a university hospital by examining the files of 49 MPS III patients who were admitted between January 1998 and December 2019.

Results: The mean age of the participants was $12.24 \pm 5.21$ years (range: 1-26). The mean age at which the patients underwent echocardiography was $6.90 \pm 4.82$ years. MPS IIIA, IIIB, IIIC, and IIID subtypes were present in $24(49.0 \%), 19(38.8 \%), 5(10.2 \%)$, and 1 ( $2.0 \%)$ patient, respectively. Among the MPS III patients who had echocardiographic evaluation $(n=44), 32$ patients $(72.7 \%)$ had pathological cardiac findings, while 12 patients $(27.3 \%)$ had normal cardiac findings on echocardiographic examination. The most common cardiac pathologies were those related to mitral valve [valve insufficiency 52.3\% ( $n=27)$, valve thickening $43.2 \%(n=25)$, and prolapse $38.6 \%(n=23)$ ]. Tricuspid insufficiency $(34.8 \%, n=8)$ was seen only in MPS IIIA. Mitral insufficiency and aortic valve thickening were significantly more common among females $(p=0.014, p=0.025$, respectively).
\end{abstract}

Conclusion: Patients with MPS III should be closely monitored for cardiac pathologies and especially mitral valve insufficiency, which are more prevalent among females.

Keywords: Mucopolysaccharidosis III, cardiac, mitral valve, tricuspid valve, sex

\section{Introduction}

Mucopolysaccharidoses (MPS) are hereditary lysosomal storage diseases in which specific enzymes that ensure the destruction of glycosaminoglycans are deficient due to genetic defects (1). In this group of diseases, a total of 11 enzyme deficiencies have been identified and examined under seven types (Type I, II, III, IVA, VI, VII, IX) (2).
Sanfilippo syndrome [MPS type III (MPS III)] is the most common type among MPS (3). In MPS III, there are four subtypes $(A, B, C, D)$, all of which are caused by a disruption in the heparan sulfate catabolism. The enzymes involved are heparan $\mathrm{N}$-sulfatase (encoded by the SCSH gene), $\alpha-\mathrm{N}$ acetyl-glucosaminidase (encoded by the NAGLU gene), acetyl $\alpha$-glucosaminidase $\mathrm{N}$-acetyltransferase (encoded by the HCSNAT gene), and N-acetylglucosamine-6-sulphatase 
(encoded by the GNS gene), respectively (4). Although very rare (1 in 70,000), each of these four subtypes has devastating effects on children (5). Additionally, MPS III shows autosomal recessive transition (6).

A clinical picture emerges where the central nervous system is affected and somatic findings are less common. The clinical course of MPS III can be discussed in three phases (7). In the first period of the disease (usually starting between 1 to 4 years of age), there is a developmental delay, especially in speech. In the second period, a marked behavioral disorder characterized by hyperactivity and sleep disturbance accompanies the picture. Usually, loss of skills acquired after ten years and a slow progression to a vegetative state are monitored eventually. Cardiovascular system disorders in MPS III disease have also been reported in the literature $(8,9)$. However, as in other MPS patients, significant clinical differences can be observed between patients.

Data in the literature regarding cardiac involvement in MPS III patients is scarce (6), the most common cardiac findings in patients with MPS were reported as thickening of the mitral valve with accompanying prolapse, insufficiency, and less frequently, stenosis (10).

\section{Materials and Methods}

We aimed to examine the cardiac involvement in MPS III disease in a University Hospital in Ankara, Turkey.

This descriptive cross-sectional study was carried out by examining the files and records in the hospital automation system of patients diagnosed with MPS III who were admitted between the $1^{\text {st }}$ lanuary 1998 and the $31^{\text {st }}$ December 2019, to Hacettepe University, Department of Pediatric Metabolism. Data were obtained from the hospital's electronic medical records (Nucleus automation system, MONAD software and counseling, Ankara, Turkey) and the patients' files. Ethical approval was obtained from the Hacettepe University Clinical Research Ethics Committee, and this study was performed in accordance with the ethical standards of the Declaration of Helsinki (CO 18/901-09).

During the study period, the files of 144 patients diagnosed with MPS were reviewed. Forty-nine patients with a diagnosis of MPS III were included in the analysis (Figure 1). MPS III was diagnosed based on the patients' specific enzyme levels and/or genetic analysis.

Echocardiographic findings were evaluated according to the subgroups of MPS III (IIIA, IIIB, IIIC, and IIID). The primary outcome variable of the study was "the thickening of the mitral valve leaflet". Secondary outcome variables

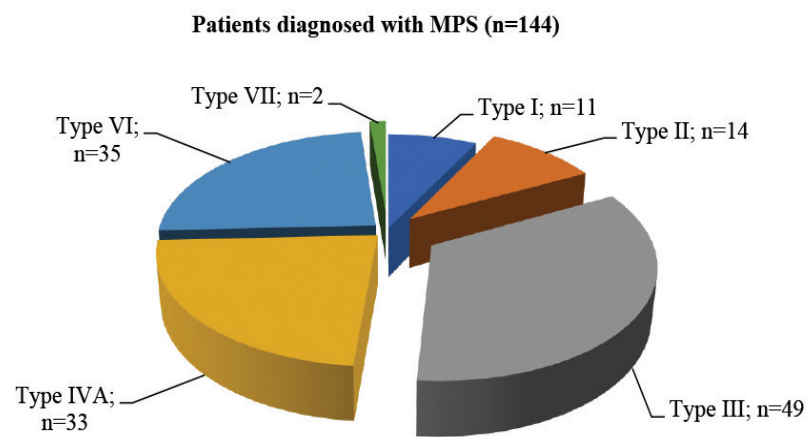

Figure 1. Diagnostic distribution of MPS patients ( $n$; number of patients) MPS: Mucopolysaccharidosis

were mitral valve prolapse, mitral valve insufficiency, tricuspid valve thickening, tricuspid valve prolapse, tricuspid insufficiency, atrial valve thickening, bicuspid aorta, atrial valve prolapse, aortic insufficiency, left ventricular ejection fraction and fractional shortening, and prognosis. The independent study variables were age and sex.

Transthoracic echocardiography was performed with Vivid E9 with an XD clear echocardiography device (GE Healthcare, General Electric Company, Wauwatosa, WI, USA). All echocardiographic studies were performed by an experienced cardiologist with a comprehensive knowledge of echocardiographic examination. Cardiac chamber quantifications were made according to pediatric guidelines (11) and the severity of valvar (aortic, mitral and tricuspid) regurgitations were defined according to the 2003 guidelines as absent, mild, moderate or severe (12).

\section{Statistical Analysis}

The Statistical Package for the Social Sciences (SPSS) (SPSS for Windows, Version 25.0, Chicago, IC, USA) program was used for statistical analysis. The results are presented as mean and standard deviation for numerical variables, and frequency and percentage for categorical data. The suitability of numerical variables to normal distribution was evaluated using skewness and kurtosis. Parametric variables were compared with the independent samples t-test for two groups and One-Way ANOVA for more than two groups. The chi-square (or Fisher's exact) test was used for comparing categorical variables. A p-value of $<0.05$ was considered sufficient for statistical significance.

\section{Results}

Out of the 144 patients diagnosed with MPS, 49 patients with a diagnosis of MPS III were included in this analysis. Twenty-six of the patients (53.1\%) were female, and twenty- 
three $(46.9 \%)$ were male. The mean age was $12.24 \pm 5.21$ years (range: 1-26) (Table I and Table II). The youngest patient with cardiac involvement (mitral insufficiency) was 1.5 years old. When the relationship between cardiac involvement and age was examined, it was found that the patients' cardiac involvement increased with age $(p=0.031)$. Nineteen $(43.2 \%)$ patients had mitral valve thickening, 32 patients $(72.7 \%)$ had pathological cardiac findings, while 12 patients (27.3\%) had normal cardiac findings on echocardiographic examination. During echocardiographic evaluation, none of the patients with cardiac involvement had severe pulmonary involvement, mechanical ventilator support, or any noninvasive ventilation support. In addition, no cardiomyopathy findings were found in any of the patients.

While making comparisons between the subtypes, there was only one patient in the $D$ subtype. Hence, this patient was not included in the comparisons. However, there was a statistically significant difference between the subtypes with respect to tricuspid insufficiency (Table III). Left ventricular ejection fraction and fractional shortening were not significantly different between the subtypes ( $F=0.363$, $p=0.698$ and $F=0.879, p=0.423$, respectively).

Concerning the comparisons made according to the prognosis, there was no significant difference in any categorical or numerical variables.

When cardiac findings were compared by sex, there was a substantial difference in some numerical and categorical variables (Table IV). Although no significant difference was found between ejection fraction values, a considerable variation was found between fractional shortening values $(t=1.718, p=0.087$ and $t=2.278, p=0.033$, respectively $)$.

\section{Discussion}

Our study demonstrated that the most common MPS III was type IIIA ( $n=24,49.0 \%)$. Thirty-two patients (72.7\%) had pathological cardiac findings, while 12 patients (27.3\%) had normal cardiac findings on echocardiographic
Table I. Description of categorical variables and dichotomous cardiac findings

\begin{tabular}{|c|c|c|c|}
\hline Variable & & $\mathbf{n}$ & $\%$ \\
\hline \multirow{2}{*}{ Sex } & Female & 26 & 53.1 \\
\hline & Male & 23 & 46.9 \\
\hline \multirow{4}{*}{ Type } & $3 \mathrm{~A}$ & 24 & 49 \\
\hline & $3 B$ & 19 & 38.8 \\
\hline & $3 C$ & 5 & 10.2 \\
\hline & $3 \mathrm{D}$ & 1 & 2 \\
\hline \multirow{2}{*}{ Echocardiography } & Absent & 5 & 10.2 \\
\hline & Present & 44 & 89.8 \\
\hline \multirow{2}{*}{ Thickened mitral valve leaflet* } & Absent & 25 & 56.8 \\
\hline & Present & 19 & 43.2 \\
\hline \multirow{2}{*}{ Mitral valve prolapse* } & Absent & 27 & 61.4 \\
\hline & Present & 17 & 38.6 \\
\hline \multirow{3}{*}{ Mitral insufficiency* } & Absent & 21 & 47.7 \\
\hline & Mild & 22 & 50 \\
\hline & Moderate & 1 & 2.3 \\
\hline \multirow{2}{*}{ Thickened tricuspid valve* } & Absent & 42 & 95.5 \\
\hline & Present & 2 & 4.5 \\
\hline \multirow{2}{*}{ Tricuspid valve prolapse* } & Absent & 43 & 97.7 \\
\hline & Present & 1 & 2.3 \\
\hline \multirow{2}{*}{ Tricuspid insufficiency* } & Absent & 36 & 81.8 \\
\hline & Mild & 8 & 18.2 \\
\hline \multirow{2}{*}{ Bicuspid aortic valve* } & Absent & 43 & 97.7 \\
\hline & Present & 1 & 2.3 \\
\hline \multirow{2}{*}{ Aortic valve thickening* } & Absent & 38 & 86.4 \\
\hline & Present & 6 & 13.6 \\
\hline \multirow{2}{*}{ Aorta valve prolapse* } & Absent & 43 & 97.7 \\
\hline & Present & 1 & 2.3 \\
\hline \multirow{3}{*}{ Aortic insufficiency* } & Absent & 37 & 84.1 \\
\hline & Mild & 6 & 13.6 \\
\hline & Moderate & 1 & 2.3 \\
\hline \multirow{3}{*}{ Prognosis } & Exitus & 6 & 12.2 \\
\hline & Follow-up & 38 & 77.6 \\
\hline & Unfollowed & 5 & 10.2 \\
\hline
\end{tabular}

Table II. Description of numeric variables and numerical cardiac findings

\begin{tabular}{|l|l|l|l|l|l|}
\hline Variable & $\mathbf{n}$ & Mean & SD & Min. & Max. \\
\hline Age & 49 & 12.24 & 5.21 & 1 & 26 \\
\hline Age at diagnosis & 49 & 5.25 & 3.00 & 0.83 & 14 \\
\hline Follow-up time & 39 & 6.43 & 5.19 & 0.17 & 19.81 \\
\hline Age at echocardiography* & 44 & 6.90 & 4.82 & 0.83 & 25 \\
\hline Ejection fraction* & 44 & 71.20 & 4.94 & 61 & 81 \\
\hline Fractional shortening* & 44 & 41.91 & 4.41 & 33 & 49 \\
\hline $\begin{array}{l}\text { *: Only 44 of the 49 patients had echocardiographic evaluation } \\
\text { SD: Standard deviation, min.: Minimum, max.: Maximum }\end{array}$ & & & & \\
\hline
\end{tabular}




\begin{tabular}{|c|c|c|c|c|c|}
\hline Variable & Subtype & Absent & Present & $\chi^{2}$ & p-value* \\
\hline \multirow{3}{*}{ Thickened mitral valve leaflet } & $3 \mathrm{~A}$ & 12 & 11 & 1,491 & 0.520 \\
\hline & $3 B$ & 11 & 7 & & \\
\hline & $3 C$ & 2 & 0 & & \\
\hline \multirow{3}{*}{ Mitral valve prolapse } & $3 \mathrm{~A}$ & 12 & 11 & 2,536 & 0.309 \\
\hline & $3 B$ & 13 & 5 & & \\
\hline & $3 C$ & 2 & 0 & & \\
\hline \multirow{3}{*}{ Mitral insufficiency } & $3 \mathrm{~A}$ & 9 & 13 & 2,154 & 0.370 \\
\hline & $3 \mathrm{~B}$ & 11 & 7 & & \\
\hline & $3 C$ & 1 & 1 & & \\
\hline \multirow{3}{*}{ Thickened tricuspid valve } & $3 \mathrm{~A}$ & 21 & 2 & 2,142 & 0.542 \\
\hline & $3 \mathrm{~B}$ & 18 & 0 & & \\
\hline & $3 C$ & 2 & 0 & & \\
\hline \multirow{3}{*}{ Tricuspid valve prolapse } & $3 \mathrm{~A}$ & 22 & 1 & 2,187 & 1.000 \\
\hline & $3 \mathrm{~B}$ & 18 & 0 & & \\
\hline & $3 C$ & 2 & 0 & & \\
\hline \multirow{3}{*}{ Tricuspid insufficiency } & $3 \mathrm{~A}$ & 15 & 8 & 8,521 & 0.010 \\
\hline & $3 \mathrm{~B}$ & 18 & 0 & & \\
\hline & $3 C$ & 2 & 0 & & \\
\hline \multirow{3}{*}{ Bicuspid aortic valve } & $3 \mathrm{~A}$ & 22 & 1 & 2,187 & 1.000 \\
\hline & $3 \mathrm{~B}$ & 18 & 0 & & \\
\hline & $3 C$ & 2 & 0 & & \\
\hline \multirow{3}{*}{ Aortic valve thickening } & $3 \mathrm{~A}$ & 18 & 5 & 2,224 & 0.414 \\
\hline & $3 \mathrm{~B}$ & 17 & 1 & & \\
\hline & $3 C$ & 2 & 0 & & \\
\hline \multirow{3}{*}{ Aorta valve prolapse } & $3 \mathrm{~A}$ & 22 & 1 & 2,187 & 1.000 \\
\hline & $3 \mathrm{~B}$ & 18 & 0 & & \\
\hline & $3 C$ & 2 & 0 & & \\
\hline \multirow{3}{*}{ Aortic insufficiency } & $3 \mathrm{~A}$ & 18 & 5 & 3,914 & 0.102 \\
\hline & $3 \mathrm{~B}$ & 17 & 1 & & \\
\hline & $3 C$ & 1 & 1 & & \\
\hline
\end{tabular}

examination. The most common cardiac pathologies were those related to mitral valve [valve insufficiency $52.3 \%$ $(n=27)$, valve thickening $43.2 \%(n=25)$, and prolapse $38.6 \%$ $(n=23)]$. Tricuspid insufficiency $(34.8 \%, n=8)$ was seen only in patients with MPS IIIA. Mitral insufficiency and aortic valve thickening were significantly more common among females.

The deposition of heparan sulfate in the tissues leads to various anomalies. In addition to the symptoms of the nervous system, in particular, they can cause respiratory, ear, nose and throat, musculoskeletal, gastroenterological, ocular, and cardiac symptoms $(13,14)$.

Followed by pneumonia, cardiorespiratory insufficiency has been reported to be the most common cause of death in MPS III syndrome (13). However, it is not clear whether the cause of cardiorespiratory insufficiency is due to respiratory problems or cardiac origin. Indeed, in our study, we could not find any evidence that cardiac pathologies increase the mortality rate. 


\begin{tabular}{|c|c|c|c|c|c|}
\hline Cardiac findings & Sex & Absent & Present & $\chi^{2}$ & p-value \\
\hline \multirow{2}{*}{ Thickened mitral valve leaflet } & Female & 11 & 13 & 2,597 & 0.135 \\
\hline & Male & 14 & 6 & & \\
\hline \multirow{2}{*}{ Mitral valve prolapse } & Female & 13 & 11 & 1,154 & 0.359 \\
\hline & Male & 14 & 6 & & \\
\hline \multirow{2}{*}{ Mitral insufficiency } & Female & 7 & 17 & 7,291 & $0.014^{*}$ \\
\hline & Male & 14 & 6 & & \\
\hline \multirow{2}{*}{ Thickened tricuspid valve } & Female & 22 & 2 & 1,746 & 0.493 \\
\hline & Male & 20 & 0 & & \\
\hline \multirow{2}{*}{ Tricuspid valve prolapse } & Female & 23 & 1 & 0.853 & 1.000 \\
\hline & Male & 20 & 0 & & \\
\hline \multirow{2}{*}{ Tricuspid insufficiency } & Female & 17 & 7 & 4,809 & 0.054 \\
\hline & Male & 19 & 1 & & \\
\hline \multirow{2}{*}{ Bicuspid aortic valve } & Female & 24 & 0 & 1,228 & 0.455 \\
\hline & Male & 19 & 1 & & \\
\hline \multirow{2}{*}{ Aortic valve thickening } & Female & 18 & 6 & 5,789 & 0.025 \\
\hline & Male & 20 & 0 & & \\
\hline \multirow{2}{*}{ Aorta valve prolapse } & Female & 23 & 1 & 0.853 & 1.000 \\
\hline & Male & 20 & 0 & & \\
\hline \multirow{2}{*}{ Aortic insufficiency } & Female & 19 & 5 & 0.957 & 0.428 \\
\hline & Male & 18 & 2 & & \\
\hline
\end{tabular}

It has been noted that the symptoms of MPS IIIA start earlier and progress faster than MPS IIIB and IIIC $(15,16)$. Furthermore, it has been reported that the IIIA subtype is more common in Sanfilippo patients than the other subtypes (17). Additionally, MPS IIID has been reported to be very rare and heterogeneous $(18,19)$. Similarly, in our study, subtype $A$ was more common with a rate of $49 \%(n=24)$, and only one case of subtype D (2\%) was detected. Similar to other studies, the subtype with the most common pathological cardiac findings was type IIIA. However, only tricuspid insufficiency was statistically significant. This finding may be due to difficulties in the analysis of rare diseases. Although it was stated that subtype $A$ was diagnosed earlier, our study did not support this data.

It has been reported that the cardiovascular system is also affected in MPS III disease. In addition, many studies have noted that cardiovascular involvement in MPS I, II, and VI patients are higher than in MPS III and IV (9, 20-22). MPS VII has not been reported on yet as it is very rare (10).

In a study conducted in Spain with 55 MPS III patients, it was reported that only one of the cases with subtype $A$ had cardiac valve involvement and four of them had mild cardiomyopathy (17). Also, in a recent study in Taiwan evaluating the echocardiographic findings of 26 patients with MPS III, the incidence of heart valve involvement was reported as $38 \%$ (8). In that study, it was reported that the most common cardiac pathology was mitral regurgitation, followed by aortic regurgitation (8).

In another recent study performed with 45 MPS III patients, the age at which the first echocardiography was performed was similar to ours. They reported a slightly lower incidence $(60 \%)$ of abnormal cardiac findings than our result (6). Similarly, in this study, the incidence of mitral valve problems were more common than other valves.

Compatible with previous studies, in our study, the most common cardiac pathology was associated with the mitral valve. In addition, tricuspid valve involvement in MPS III patients observed in our study is remarkable and had not been reported in previous studies. Furthermore, another important finding of our research is that features of cardiac involvement may differ by sex. To the best of our knowledge, our study is the first to mention this significant difference. 


\section{Study Limitations}

This is a retrospective study based on hospital records. Thus, the reliability of the recorded data might be a concern in this type of research. However, we consider that the recorded information is reliable since the cardiac evaluations were performed by an experienced cardiologist with a comprehensive knowledge concerning echocardiographic examination. Despite repeated follow-ups of the patients, the evaluation of all patients was based on their firstrecorded echocardiographic findings. Since MPS is a progressive disease, it would be of value to observe the cardiac involvement changes over time.

\section{Conclusion}

According to our results, the majority of patients with MPS III have cardiac involvement and it is most commonly related to the mitral valve, therefore these patients should be closely monitored by echocardiography. Tricuspid insufficiency was seen only in those patients with MPS IIIA. Mitral insufficiency and aortic valve thickening were significantly more common among females.

MPS IIIA subtype and female sex may increase the risk of cardiac pathologies. Although verification by future studies is warranted, considering the relatively large number of patients in our study, our results are important.

\section{Ethics}

Ethics Committee Approval: Ethical approval was obtained from the Hacettepe University Clinical Research Ethics Committee, and this study was performed in accordance with the ethical standards of the Declaration of Helsinki (CO 18/901-09).

Informed Consent: Retrospective study.

Peer-review: Internally and externally peer-reviewed.

\section{Authorship Contributions}

Medical Practice: B.B.G., E.A., H.S.S., Concept: A.T., T.C., Design: B.B.G., H.S.S., Data Collection or Processing: E.A., D.A., Analysis or Interpretation: E.A., D.A., A.T., T.C., H.S.S., Literature Search: T.C., A.D., H.S.S., Writing: B.B.G.

Conflict of Interest: No conflict of interest was declared by the authors.

Financial Disclosure: The authors declared that this study received no financial support.

\section{References}

1. Zhou J, Lin J, Leung WT, Wang L. A basic understanding of mucopolysaccharidosis: Incidence, clinical features, diagnosis, and management. Intractable Rare Dis Res 2020; 9:1-9.
2. Coutinho MF, Lacerda L, Alves S. Glycosaminoglycan storage disorders: a review. Biochem Res Int 2012;2012:471325. doi: $10.1155 / 2012 / 471325$

3. Osipova LA, Kuzenkova LM, Namazova-Baranova LS, Gevorkyan AK, Podkletnova TV, Vashakmadze ND. [Sanfilippo Syndrome]. Vestn Ross Akad Med Nauk 2015: 419-27.

4. Gaffke L, Pierzynowska K, Piotrowska E, Węgrzyn G. How close are we to therapies for Sanfilippo disease? Metab Brain Dis 2018; 33:1-10.

5. Zelei T, Csetneki K, Vokó Z, Siffel C. Epidemiology of Sanfilippo syndrome: results of a systematic literature review. Orphanet I Rare Dis 2018; 13:53.

6. Wilhelm CM, Truxal KV, McBride KL, Kovalchin JP, Flanigan KM. Natural history of echocardiographic abnormalities in mucopolysaccharidosis III. Mol Genet Metab 2018; 124:131-4.

7. Valstar MJ, Ruijter G), van Diggelen OP, Poorthuis BJ, Wijburg FA. Sanfilippo syndrome: a mini-review. I Inherit Metab Dis 2008; 31:240-52.

8. Lin HY, Chen MR, Lin SM, et al. Cardiac characteristics and natural progression in Taiwanese patients with mucopolysaccharidosis III. Orphanet / Rare Dis 2019; 14:140.

9. Leal GN, de Paula AC, Leone C, Kim CA. Echocardiographic study of paediatric patients with mucopolysaccharidosis. Cardiol Young 2010; 20:254-61.

10. Braunlin EA, Harmatz PR, Scarpa M, et al. Cardiac disease in patients with mucopolysaccharidosis: presentation, diagnosis and management. J Inherit Metab Dis 2011; 34:1183-97.

11. Lopez L, Colan SD, Frommelt PC, et al. Recommendations for quantification methods during the performance of a pediatric echocardiogram: a report from the pediatric measurements writing group of the American Society of Echocardiography Pediatric and Congenital Heart Disease Council. I Am Soc Echocardiogr 2010; 23:465-95; quiz 576-7.

12. ZoghbiWA, Enriquez-Sarano M, FosterE, etal. Recommendations for evaluation of the severity of native valvular regurgitation with two-dimensional and Doppler echocardiography. I Am Soc Echocardiogr 2003; 16:777-802.

13. Lavery C, Hendriksz C), Jones SA. Mortality in patients with Sanfilippo syndrome. Orphanet I Rare Dis 2017; 12:168.

14. Mitchell ), Berger Kl, Borgo A, et al. Unique medical issues in adult patients with mucopolysaccharidoses. Eur I Intern Med 2016; 34:2-10.

15. Meyer A, Kossow K, Gal A, et al. Scoring evaluation of the natural course of mucopolysaccharidosis type IIIA (Sanfilippo syndrome type A). Pediatrics 2007; 120:e1255-61. doi: 10.1542/ peds.2007-0282.

16. Ruijter G), Valstar MJ, van de Kamp JM, et al. Clinical and genetic spectrum of Sanfilippo type C (MPS IIIC) disease in The Netherlands. Mol Genet Metab 2008; 93:104-11.

17. Delgadillo V, O'Callaghan Mdel M, Gort L, Coll MJ, Pineda M. Natural history of sanfilippo syndrome in Spain. Orphanet / Rare Dis 2013; 8:189.

18. Jansen AC, Cao H, Kaplan P, et al. Sanfilippo syndrome type D: natural history and identification of 3 novel mutations in the GNS Gene. Arch Neurol 2007; 64:1629-34.

19. Beesley CE, Burke D, Jackson M, Vellodi A, Winchester BG, Young EP. Sanfilippo syndrome type D: identification of the first 
mutation in the $\mathrm{N}$-acetylglucosamine-6-sulphatase gene. J Med Genet 2003; 40:192-4.

20. Gross DM, Williams JC, Caprioli C, Dominguez B, Howell RR. Echocardiographic abnormalities in the mucopolysaccharide storage diseases. Am J Cardiol 1988; 61:170-6.
21. Chen MR, Lin SP, Hwang HK, Yu CH. Cardiovascular changes in mucopolysaccharidoses in Taiwan. Acta Cardiol 2005; 60:51-3.

22. Fesslová V, Corti P, Sersale G, et al. The natural course and the impact of therapies of cardiac involvement in the mucopolysaccharidoses. Cardiol Young 2009; 19:170-8. 